\title{
Variationen von Sprache
}

Gesamtüberblick: Die sprachliche Kommunikation ist geprägt durch die Vielfalt an Varianten, die existieren, um Gedanken und Beobachtungen sprachlich auszudrücken. Ein Instrument zur sprachlichen Variation von Textaufgaben im Mathematikunterricht sollte sich an den Veränderungen von Sprache in der Nutzung orientieren. Daher ist das Ziel dieses Kapitels die bedeutsamen Konzepte von Variationen von Sprache zu beschreiben.

Die Flexibilität der Sprache lässt sich durch sprachliche Variationen darstellen, die sich grundlegend in Dialekte und Register unterscheiden lassen (Abschnitt 4.1). Aufgrund des vorliegenden Forschungsfokus ist das Konzept des Registers relevant, da das Register Variationen theoretisch erklärt, die beispielsweise aufgrund von institutionellen oder fachspezifischen Gründen auftreten (Abschnitt 4.2). Maßgeblich für die Betrachtung des Registerbegriffs ist die Klärung des Begriffs durch eine Definition und die Darstellung unterschiedlicher Akzentuierungen (Abschnitt 4.2.1). In der mathematikdidaktischen Forschungsliteratur werden typischerweise drei unterschiedliche Register betrachtet, die für den Mathematikunterricht als relevant erachtet werden. Im Mathematikunterricht wird Sprache gebraucht, die ebenfalls im Alltag genutzt wird und das Register der Alltagssprache darstellt (Abschnitt 4.2.2). Die Institution Schule entwickelt eine Sprache der Vermittlung, die den Zweck erfüllt, für didaktische Prozesse genutzt zu werden, und die als Register der Schulsprache bezeichnet werden kann (Abschnitt 4.2.3). Neben der für didaktische Zwecke genutzten Sprache wird in der Institution Schule außerdem eine habitualisierte Sprache für die Vermittlung

Elektronisches Zusatzmaterial Die elektronische Version dieses Kapitels enthält Zusatzmaterial, das berechtigten Benutzern zur Verfügung steht https://doi.org/10.1007/978-3-658-33003-3_4.

D. Bednorz, Sprachliche Variationen von mathematischen Textaufgaben, Bielefelder Schriften zur Didaktik der Mathematik 5, https://doi.org/10.1007/978-3-658-33003-3_4 
verwendet, die u. a. aus inhaltlichen Gründen verwendet und als bildungssprachliches Register charakterisiert wird (Abschnitt 4.2.4). Für den Gegenstandbereich der Mathematik ergeben sich spezifische Merkmale, die sich in der Verwendung einer Sprachvariation äußern, die als mathematisches (fachsprachliches) Register bezeichnet werden kann (Abschnitt 4.2.5). Da die Verwendung von Sprache kein statischer Prozess ist, gilt auch für die Verwendung von Registern, dass sich Änderungen ergeben, die mit Veränderungen von Situationen in Beziehungen stehen (Abschnitt 4.3). Registervariationen sind ein empirisches Phänomen. Für die Analyse von Registervariationen haben sich empirische Methoden entwickelt, die insbesondere mit quantitativen Verfahren mit computerbasierter Ermittlung die sprachlichen Merkmale (korpusbasierte Ansätze) ermitteln (Abschnitt 4.4). Durch die empirische Ermittlung von sprachlichen Merkmalen ergibt sich unterschiedliches Akzentuieren der Analyse (Abschnitt 4.5). So kann mit den computerbasierten Verfahren die Häufigkeiten von einzelnen sprachlichen Merkmalen festgestellt werden (Abschnitt 4.5.1). Außerdem besteht die Möglichkeit, das gemeinsame Vorkommen von sprachlichen Merkmalen festzustellen (Abschnitt 4.5.2). Zur Analyse des gemeinsamen Vorkommens von sprachlichen Merkmalen ergeben sich spezifische Verfahren, die als multivariate Verfahren bezeichnet werden (Abschnitt 4.5.3).

\subsection{Formen von sprachlichen Variationen}

Sprache ist im stetigen Wandel. Dies demonstrierten die angeführten Beispiele in Abschnitt 3.4, die die Vielfalt an unterschiedlichen Variationen sprachlicher Ausdrucksmöglichkeiten für den Mathematikunterricht exemplarisch zeigen. Die Unterschiede in der Verwendung von Sprache sind so prägnant in Gestalt und Vorkommen, dass bereits Freudenthal (1983) auf die Unterschiede aufmerksam machte und beschreibt, dass jeder Einzelne verschiedene Formen von Sprache verwendet, und dabei pointiert, dass keine zwei Personen dieselbe Sprache sprechen und es maßgeblich vom Ort, Dialekt, der Bildungssprache und der mathematischen Sprache abhängt, wie Sprache genutzt wird. Die durch Freudenthal (1983) ohne direkten linguistischen Bezug dargestellten Bedingungen deuten darauf hin, dass sich sprachliche Phänomene je nach Spezifika der Sprachnutzung ausbilden. Nach Ferguson (1994) sind es insbesondere sich wiederholende Ereignisse, die im Verlauf von Menschen mit ähnlichen sprachlichen Strukturen assoziiert und die in den Ereignissen wiederverwendet werden: 
[a] communication situation that recurs regularly in a society (in terms of participants, setting, communicative functions, and so forth) will tend over time to develop identifying markers of language structure and language use, different from the language of other communication situations. People participating in recurrent communication situations tend to develop similar vocabularies, similar features of intonation, and characteristic (S. 20).

In Hinblick darauf erarbeitete Ferguson (1994) eine Arbeitsdefinition für Sprachvariationen, indem er sprachliche Variationen als mehr oder weniger deutlich abgrenzbare sprachliche Einheiten betrachtet, die, je deutlicher sie ausfallen, desto vorteilhafter als Analysegegenstand genutzt werden können:

Sets of identifying markers [...] vary greatly in the degree of cohesiveness they show as systems and the sharpness of the boundaries between them; the more cohesive the systems, the sharper the boundaries, and the more they are perceived by the participants as separate entities, the more useful it is to analyze them as language varieties (S. 23).

Zur Klassifikation solcher Sprachvariationen unterscheidet Ferguson (1994) vier Kategorien, durch die Sprache durch Verwendung variieren kann: Dialekt, Register, Genre und Konversationen. Häufig existiert in der Forschungsliteratur zum Thema sprachliche Variationen jedoch nur eine Unterscheidung in zwei Kategorien: Dialekt und Register (Biber, 2006; Finegan \& Biber, 2001; Halliday, 1978, 2003a, 2014a; Ure \& Ellis, 2014).

Die beiden Hauptkategorien von sprachlichen Variationen lassen sich begrifflich beschreiben. Sprachliche Variationen, deren Ursprung einer Gruppe von Sprechern zuzuordnen ist und die sich aufgrund unterschiedlicher lokaler und sozialer Varietäten in derselben Sprache ausbilden, werden als Dialekt definiert (Halliday, 1978). Ferguson (1994) bezeichnet einen Dialekt als die sprachliche Variation, die eine Person spricht, determiniert durch das, was sie ist. Ein Register wird als Variation einer Sprache aufgrund des Sprachgebrauchs und der Sprachhandlung definiert (Halliday, 1978). Nach Ferguson (1994) bezeichnet ein Register die sprachliche Variation, die eine Person spricht, determiniert durch das, was sie gerade tut. Da für den Forschungsgegenstand der vorliegenden Arbeit nur der Begriff des Registers als sprachliche Variation relevant ist, wird der Begriff des Dialekts nicht mehr betrachtet.

Neben der Beschreibung von sprachlichen Variationen ist das Register als eine Form von sprachlicher Variation bedeutsam für das Lehren und Lernen. Laut Ure und Ellis (2014) demonstriert die Breite des erfolgreichen Umgangs mit Registern die Spracherfahrung. In dieser Hinsicht wird die Beherrschung von 
Registern in besonderem Maß für Lehr- und Lernprozesse im Mathematikunterricht relevant. Der Aspekt von Sprache als Lernvoraussetzung und -hindernis in Abschnitt 2.4.3 wird damit durch die Betrachtung von sprachlichen Variationen unterstrichen. So gilt es für Lernende im Mathematikunterricht, kompetent mit den unterschiedlichen Registern umzugehen; dies gelingt umso besser, desto mehr Spracherfahrung vorhanden ist. Nachfolgend soll aus diesem Grund neben einer weitreichenden Diskussion der Definition von Registern dargestellt werden, welche unterschiedlichen Register im Mathematikunterricht vorkommen und wie sich diese auszeichnen.

\subsection{Register}

Das Konzept des Registers beschreibt relevante Aspekte von sprachlichen Veränderungen für das Lernen und Lehren im Mathematikunterricht. Um den Einfluss der Register für den Mathematikunterricht deutlich zu machen, ist eine Analyse der unterschiedlichen Registern, die im Mathematikunterricht vorkommen, und der sprachlichen Merkmale, die mit diesen Registern verbunden sind, zu beschreiben.

Überblick (Abschnitt 4.2): In der Diskussion des Registers ergeben sich neben der in Abschnitt 4.1 dargestellten Beschreibung unterschiedliche Perspektiven, den Begriff des Registers auszulegen (Abschnitt 4.2.1). Für den Mathematikunterricht ergeben sich unterschiedliche Register, die typischerweise im Mathematikunterricht vorkommen können und sich durch spezifische Merkmale auszeichnen. Hierzu zählen Register der Alltagssprache als generealistische sprachliche Variation (Abschnitt 4.2.2). Des Weiteren ist die Schulsprache als Register für den Unterricht von Mathematik relevant, da hier besonders die Sprache akzentuiert wird, die zur didaktischen Vermittlung genutzt wird (Abschnitt 4.2.3). Daneben ist das bildungssprachliche Register ähnlich wie das schulsprachliche Register aufgrund von Vermittlungsaspekten für den Mathematikunterricht bedeutsam (Abschnitt 4.2.4). Aufgrund der fachlichen Gestaltung ergeben sich für den Mathematikunterricht Spezifika der Fachsprache Mathematik, die im Mathematikunterricht durch das mathematische Register vorkommen (Abschnitt 4.2.5). 


\subsubsection{Deutung des Registerbegriffs}

Der Registerbegriff wurde von Reid (1956) eingeführt, um ein Konzept zu entwickeln, das Sprachvariationen korrespondierend mit Situationsvariationen vereinigt. Dieses Variationskonzept beschreibt den Zusammenhang von veränderlichen Sprachstrukturen und den Kontext der Situation (vgl. Abschnitt 3.3.2). Neben den unterschiedlichen Auslegungsvarianten des Registerbegriffs definiert Biber (2006) Register als Oberbegriff für jegliche Variation, die mit einem bestimmten Kontext der Situation oder Zweck assoziiert ist.

In der Diskussion rund um den Registerbegriff ergeben sich unterschiedliche Interpretationsmöglichkeiten. In einer frühen Auslegung des Registerbegriffs definieren Halliday und Hasan (1989) Register als Konfiguration von Bedeutungen im Zusammenhang mit situativen Konfigurationen, die mit den drei Ebenen des Kontextes einer Situation in Verbindung stehen, die in Abschnitt 3.3.3 beschrieben wurden:

a configuration of meanings that are typically associated with a particular situational configuration of field, mode, and tenor (S. 38-39).

Ure und Ellis (2014) bezeichnen Register als ein konventionalisiertes soziales Phänomen innerhalb von Sprachgemeinschaften, das zur Bildung von regulären Sprachmustern in bestimmten Situationen führt. Die regulären Sprachmuster bezeichnet Halliday (2014a) als besondere Zusammenstellung von systemischen Wahrscheinlichkeiten (Halliday, 2014a). Halliday (2005) nutzt bei seiner Beschreibung für das Register häufig probabilistische Aussagen zur Definition eines Registers:

A register is a tendency to select certain combinations of meanings with certain frequencies [...] (S. 66).

Dabei beschreibt ein Register eine Auswahl an semantischen Ressourcen, die Mitglieder einer Sprachgemeinschaft typischerweise mit einem Situationstypus mit gewissen Häufigkeiten identifizieren. In der Diskussion um Register wird dahingehend der Aspekt hervorgehoben, dass für bestimmte Register spezifische sprachliche Merkmale beschrieben werden können, deren Häufigkeit mit bestimmten Situationen in Verbindung stehen.

Trotz der Wahrscheinlichkeitsaussagen durch die begriffliche Beschreibung von Registern in Form von Möglichkeiten, Optionen, Potenzialen und Frequenzen weist Halliday (2014b) auf die Alltäglichkeit des Phänomens Register hin: 
[...] but the existence of registers is a fact of everyday experience - speakers have no difficulty in recognizing the semantic options and combinations of options that are at risk under particular environmental conditions. Since these options are realized in the form of grammar and vocabulary, the register is recognizable as a particular selection of words and structures. But it is defined in terms of meanings, it is not an aggregate of conventional forms of expression superposed on some underlying content by social factors of one kind or another. It is the selection of meanings that constitutes the variety to which a text belongs (S. 267).

Die von Halliday (2014b) beschriebene Alltäglichkeit des Phänomens des Registers lässt sich als konstruktiver Akt der Sprachnutzung und -handlung deuten. Nach Ure und Ellis (2014) repräsentieren die variantenreichen Ausprägungen von unterschiedlichen Registern die damit korrespondierende Erfahrungs- und Bedeutungskonstruktion einer Gesellschaft. Dahingehend verweist Biber (2006) darauf, dass Register auf jeder Ebene der Generalität betrachtet werden können. So existieren tendenziell eher generalistische Register wie das alltagssprachliche Register (vgl. Abschnitt 4.2.2), aber auch hochspezielle Register wie das mathematische Register (vgl. Abschnitt 4.2.5). Dabei korrespondiert die sprachliche Reichweite des Registers mit der Reichweite der Situation, in der die Sprache genutzt werden kann (Ure \& Ellis, 2014).

Für die Interpretation des Registerbegriffs ergeben sich weitreichende Konsequenzen. So kann, wenn in einer Sprachgemeinschaft Communitys existieren, die sich durch die Festigung von Aktivitäten und Sprachtraditionen in unterschiedlichen situativen Parametern unterscheiden (vgl. Abschnitt 3.3.2), aus dem Registerbegriff prognostiziert werden, dass diese Communitys ein unterschiedliches Sprach- bzw. Registerrepertoire nutzen werden. Durch die Nutzung unterschiedlicher Registermuster wird innerhalb einer Sprachgemeinschaft symbolisiert, dass sich die Sprecher einer spezifischen sozialen Situation bewusst sind. Durch die bewusste Nutzung oder Vermeidung von Registern kann ein Sprecher versuchen, bewusst die Verschiebung des Kontextes der Situation zu realisieren (Ure \& Ellis, 2014).

\subsubsection{Alltagssprache}

Insbesondere in der Diskussion über Register in der Fachdidaktik und Fachsprachenforschung wird häufig neben der Unterscheidung zwischen bildungssprachlichen und fachsprachlichen Registern zusätzlich das alltagssprachliche Register unterschieden (Gogolin \& Lange, 2011; Meyer \& Prediger, 2012; Rincke, 2010; Vollmer \& Thürmann, 2010, 2013). 
Häufig bleibt dabei unklar, was Alltagssprache bedeutet und welche Relevanz dieser Begriff hat. Für Alltagssprache ergeben sich viele synonyme Begriffsverwendungen, beispielsweise: Gemeinsprache, Umgangssprache, Nationalsprache, Landessprache, Volkssprache oder Standardsprache (Hoffmann, 2008; Trabant, 1983). ${ }^{1}$ So kann Alltagssprache auch als diejenige Sprache bestimmt werden, über die die Mehrheit der Sprachgemeinschaft verfügt, und mit der die allgemeine Kommunikation erst ermöglicht wird (Hoffmann, 2008).

Unter der Perspektive des Registers ist das alltagsprachliche Register definierbar, in dem betrachtet wird, welcher Kontext der Situation für die Kommunikation entscheidend ist. Insbesondere wird Alltagssprache dort deutlich, wo sie in Kontrast zur Fachsprache steht (Trabant, 1983) (vgl. Abschnitt 4.2.5). Ammon (1977) bietet dahingehend eine begriffliche Festigung, was unter dem alltagssprachlichen Register verstanden werden kann, indem der Kontrast zur Fachsprache gebildet wird und typische Aktivitäten, die der fachsprachlichen Sphäre zugeordnet, und Aktivitäten, die alltäglich sind, gegenübergestellt werden:

Fachsprache und Gemeinsprache bilden ein komplementäres Begriffspaar. Als Gemeinsprache werden diejenigen Teile des Gesamtsprachrepertoires der Gesellschaft bezeichnet, die in den für alle Gesellschaftsmitglieder einigermaßen ähnlichen Lebensbereichen gebräuchlich sind und sich auf allgemeinbekannte Gegenstände, Sachverhalte und Vorstellungen beziehen. Differenziert ist die Gesellschaft aber vor allem in der Arbeitssphäre. In ihr ist folglich die Fachsprache hauptsächlich verankert. Für alle Gesellschaftsmitglieder einigermaßen gleichartige Bereiche finden sich dagegen primär in der Konsumtionssphäre, auf die sich die Gemeinsprache in erster Linie bezieht. Allerdings gibt es auch Spezialisierungen und Differenzierungen in der Konsumtionssphäre, beispielsweise die Vielfalt der Hobbywelt. Bei den dafür spezifischen Sprachinhalten (S. 29).

Durch die Referenz auf Fachsprache bildet Ammon (1977) eine grundlegende Beschreibung für das Phänomen Alltagssprache. Sie ist diejenige Sprache, die dadurch beschrieben werden kann, dass sie am geringsten durch situative Parameter fragmentiert ist. Damit sind Situationen gemeint, die für eine breite gesellschaftliche Schicht zugänglich sind. Trabant (1983) formuliert eine Definition der Alltagssprache, die ebenfalls auf dem Gegensatz zwischen Fachsprache und Alltagssprache basiert, spezifiziert jedoch dahingehend, dass nicht die Arbeitssphäre in der Betrachtung der Fachsprache gesehen wird, sondern das durch die Fachexpertise entwickelte spezielle sprachliche Repertoire in Form von Fachbegriffen und Nomenklaturen, die der weiteren Welt- und Bedeutungserschließung dienen:

${ }^{1}$ Der Begriff Alltagssprache wird nachfolgend für alle diese synonymen Begriffe verwendet. 
Die Opposition von Gemeinsprache und Fachsprache basiert im Wesentlichen auf der Erfahrung des Unterschieds zwischen allgemein bekannten und nicht allgemein bekannten Sachen: Die Gemeinsprache deckt alle allgemein bekannten Sachen mit Wörtern ab, die Fachsprache dagegen benennt solche Sachen, die nicht allgemein bekannt sind, sondern nur von Fachleuten, Experten gewußt werden [...] [(Alltags)]Sprache insgesamt wird dabei als eine enger oder weiter auf die Welt ausgedehnte bzw. mehr oder minder fein differenzierte Nomenklatur verstanden, deren gemeinsamer Kern, der common core, das allgemeine, nicht spezialistisches Wissen über die Welt sprachlich repräsentiert (S. 29).

Trabant (1983) forciert in seiner Definition der Alltags- und Fachsprache die Funktion der unterschiedlichen Registervarianten. Beide dienen als sprachliche Ressourcen. Die Fachsprache dient als sprachliche Ressource zur Welt- und Bedeutungserschließung für eine Expertengruppe und die Alltagssprache als gemeinsamer Kern einer Sprachgemeinschaft, um allgemeine Situationen, die eine Sprachgemeinschaft teilen, durch Sprache zu erschließen. Beispiele für das alltagssprachliche Register können die Nutzung von Sprache im Kontext der Familie, in der Öffentlichkeit, in der Heimatstadt, im Urlaub und beim Einkaufen sein.

In Hinblick auf das Lehren und Lernen von Mathematik wird das alltagssprachliche Register häufig in Beziehung mit den Basic Interpersonal Communication Skills (BICS) gebracht (vgl. Abschnitt 2.4.2) (Cummins, 2017; Vollmer \& Thürmann, 2010, 2013). Wie in Abschnitt 2.4.2 erläutert, handelt es sich bei BICS um sprachliche Fähigkeiten, die genutzt werden, um in Alltagssituationen kommunizieren zu können (Cummins, 2017). BICS zeichnen sich damit am deutlichsten durch einen geringen sprachlichen Grad einer Fragmentierung und eine geringe Komplexität sowie generalisierbaren Aussagen aus.

Sprachliche Merkmale des alltagssprachlichen Registers: Gogolin und Lange (2011) beschreiben das alltagssprachliche Register dahingehend, dass die Verwendung des Registers auf eine alltagssprachliche Situation bezogen ist, in der sich die Sprechenden auf einen gemeinsamen Kontext beziehen können. Die damit beschriebene Deutung entspricht den erläuterten Beschreibungen in den Definitionen und in Hinblick auf die Interpretation von BICS, als grundlegende sprachliche Fähigkeit zu kommunizieren. Ausgehend von dieser Ausdeutung des Begriffs leiten Gogolin und Lange (2011) sprachliche Merkmale dieses Registers $\mathrm{ab}$, die beispielhaft für die Verwendung sind. Als Merkmale werden deiktische Sprach- und Deutungsmittel und die Kommunikation durch Sätze, die grammatisch unvollständig sind, genannt. 


\subsubsection{Schulsprache}

Lehrkräfte greifen aktiv in den Sprachgebrauch von Lernenden ein und normieren damit das, was im Unterricht gesagt wird (Feilke, 2012b). Die Fähigkeiten, Kenntnisse und Erfahrungen des alltagssprachlichen Registers reichen für Lernende im institutionalisierten Rahmen der Schule nicht aus, denn die Voraussetzungen der Kommunikation im Unterricht gehen über die Fertigkeiten der Verwendung des alltagssprachlichen Registers hinaus (vgl. Abschnitt 2.4.2 und Abschnitt 2.4.3) (Feilke, 2012a; Schleppegrell, 2012). Normiert wird auf Grundlage der Spracherwartungen, die schulisch-institutionalisiert festgeschrieben werden (Feilke, 2012b).

Feilke (2012b) betrachtet die Schulsprache als ein spezifisch auf die situativen Bedingungen der Institution Schule gerichtetes Register. Unter diesem Aspekt wird Schulsprache dahingehend bestimmt, dass sie in einer Wechselbeziehung mit den Aktivitäten und Gegenständen des Unterrichts betrachtet wird. Folglich wird die Sprache der Schule nicht nur im Unterricht genutzt, sondern es findet institutionell die didaktische Konstruktion des sprachlichen Registers statt. Durch die didaktische Konstruktion, die durch die Intention der Vermittlung und des Lernens geprägt ist, wird eine spezifische für den Unterricht entwickelte und genutzte Sprache geschaffen. Damit dient das schulsprachliche Register als Basis für das Lehren und Lernen im Unterricht (Vollmer \& Thürmann, 2010). Dahingehend definieren Vollmer und Thürmann (2010) Schulsprache besonders im Aspekt der in Abschnitt 2.3.1 erörterten kognitiven Funktion:

[...] [Schulsprache ist] Ausdruck jener sprachlichen bzw. kommunikativen Anforderungen in fachlichen Lernkontexten, hinter denen sich komplexe Herausforderungen in der Verwendung von Sprache als kognitivem Werkzeug verbergen (S. 110).

Die Charakterisierung der Schulsprache beschreibt zum einen die Assoziation der sprachlichen Anforderungen mit fachlichen Kontexten, dementsprechend die in Abschnitt 3.3 und Abschnitt 4.2.1 erläuterte Relevanz zwischen Sprache und Kontext, und zum anderen die komplexen Herausforderungen, die mit dem schulsprachlichen Register einhergehen. Komplex sind diese Herausforderungen, da sie curricular wenig bis nicht sichtbar sind und für Lernende eine Hürde in der Bildungsbiografie darstellen können. Cathomas (2007) betrachtet die Problematisierung der Schulsprache als Art heimlicher Lehrplan, indem er Kritik daran übt, wie sprachliche Lernziele in der Schule konstruiert werden und welchen Fokus sie einnehmen: 
Die Schule wollte für den außerschulischen Sprachalltag vorbereiten, hat dabei aber übersehen, dass sie selber eine eigene Sprachumgebung mit eigenen Regeln des Spracherfolges ist. Die Schule kann und muss nicht für den Alltag vorbereiten, dafür ist der Alltag besser geeignet. Sie hat genug damit zu tun, die Lernenden sprachlich für sich selber, für den Lebensraum Schule vorzubereiten (S. 110).

Das schulsprachliche Register, das dominant für die Vermittlung des Stoffs im Unterricht zuständig ist, muss dahingehend stärker in das Zentrum der Vermittlung rücken. Nur mit sprachlichem Lernen, zu dessen Gegenstand das schulsprachliche Register gehört, wird fachliches Lernen möglich. Daher können auch mathematische Inhalte ohne hinreichende Beherrschung des schulsprachlichen Registers nur unzureichend verstanden und gelernt werden, da sich im Verlauf der Didaktisierung der Vermittlung mathematischer Inhalte spezifische sprachliche Merkmale und Merkmalsmuster abgebildet haben, die es zu beherrschen gilt.

Sprachliche Merkmale des schulsprachlichen Registers: Auch für das schulsprachliche Register bilden sich Spezifika des Sprachgebrauchs ab. Darunter zählen beispielsweise besondere grammatische Konstruktionstypen, u. a. ein gewisser Grad an Literarität, Kenntnisse der Schriftsprachlichkeit und besondere sprachliche Eigenschaften bei der Unterscheidung von Registern (Genre, Texttypen) (Cathomas, 2007; Feilke, 2012b; Schleppegrell, 2001).

Im Vergleich zum alltagssprachlichen Register (vgl. Abschnitt 4.2.2) kann das schulsprachliche Register nach Vollmer und Thürmann (2010, S. 109) durch Adjektive wie ,prägnant, präzise, vollständig, komplex, strukturiert, objektiv, distant, emotionsfrei, eindeutig, situationsungebunden und dekontextualisiert“" beschrieben werden; sie ergeben sich aus der Verwendung der sprachlichen Mittel, deren Auswahl stets von den schulischen Bestimmungsfaktoren abhängig sind.

\subsubsection{Bildungssprache}

Abgrenzung zur Schulsprache: In Hinblick auf das in Abschnitt 4.2.3 vorgestellte schulsprachliche Register ist das Verhältnis zwischen Schulsprache und Bildungssprache zu bestimmen, da sich die Bildungssprache ebenfalls stark auf die Institution Schule bezieht.

Häufig finden beide Begriffe in der Analyse und Bewertung von schulischen Lehr-Lern-Prozessen Verwendung (Morek \& Heller, 2012). So werden unter Begriffen wie Bildungssprache, Academic Language oder Cognitive Academic 
Language Proficiency (CALP) häufig Aspekte des schulsprachlichen und bildungssprachlichen Registers subsummiert (Cummins, 1979, 1986, 2017; Morek \& Heller, 2012; Schleppegrell, 2006). Für einzelne Analysen von Sprache im Lehr-Lern-Kontext ist dagegen eine Unterscheidung zwischen Schul- und Bildungssprache sinnvoll (Schleppegrell, 2001, 2004).

Nach Feilke (2012a) lässt sich der Unterschied zwischen Schul- und Bildungssprache damit darstellen, dass Schulsprache als Aspekt bzw. Ausschnitt der Bildungssprache betrachtet wird und sich die Schulsprache eine Schnittmenge an sprachlichen Merkmalen mit der Bildungssprache teilt. Die Schulsprache ist dahingehend eingegrenzt, dass mit diesem Begriff nur sprachliche Mittel betrachtet werden, die sich rein auf den Kontext Schule beziehen. Die Bildungssprache ist im Vergleich zur Schulsprache durch viel allgemeinere Sprachhandlungen bestimmt, die vom didaktischen Kontext der Schule und deren schultypischer Entwicklung spezifischer sprachlicher Traditionen und Normen entrückt sind.

Die Bildungssprache entwickelte lexikalische und grammatische Formen, die sich durch historische Entwicklungen, beispielsweise durch habituelle Distinktionsmechanismen und die Bildung von wissenschaftlichen Domänen zum Bedeutungs- und Erkenntnisgewinn, internalisiert haben (Feilke, 2012a; Morek \& Heller, 2012). Gleichzeitig bedeutet dies, dass Bildungssprache nicht zwingend funktional für die Vermittlung von Inhalten ist; die Nutzung wird auf institutioneller, unter Umständen aufgrund tradierter Gründe, und inhaltlicher Ebene begründet (Morek \& Heller, 2012; Schleppegrell, 2012). Obwohl das bildungssprachliche Register nicht zwangsläufig funktional für das Lernen aus didaktischer Perspektive ist, wird das bildungssprachliche Register aus normativer Sicht von Lernenden für den Schulerfolg verlangt (vgl. Abschnitt 2.4.3) (Gogolin \& Lange, 2011).

Sprachliche Merkmale des bildungssprachlichen Registers: Situationen, die Bildungssprache benötigen, sind gegebenen, wenn verallgemeinerte Bedeutungskonstruktionen auf Basis von präzise gewählten sprachlichen Mittel angestrebt werden (Gogolin \& Lange, 2011). Da das Lernen der Bildungssprache in die Erkenntnis- und Bedeutungskonstruktion integriert ist, benötigen Lernende in der Schule die Möglichkeit, ein Bewusstsein für die Bildungssprache zu erlangen und die Bildungssprache über sprachliche Aktivitäten zu nutzen (Schleppegrell, 2012).

Nach Gogolin und Lange (2011) orientiert sich das Register Bildungssprache konzeptuell eher an schriftsprachlichen Sprachhandlungsmustern. Dabei hat das bildungssprachliche Register eine hohe Bedeutung für alle Handlungen, die mit Lehr- Lernprozessen assoziiert sind, beispielsweise in Aufgaben, Lehrwerken, Unterrichtsmaterialien, Leistungsüberprüfungen und Unterrichtsgesprächen. 
Sprachliche Spezifika sind u. a. die verwendete Terminologie, also die Verwendung eines bestimmten Wortschatzes, aber auch weitere lexikalische und grammatische Merkmale (vgl. Anhang). Neben der in Anhang zu findenden Auflistung ergeben sich weitere Bestimmungen der lexikalischen und grammatischen Merkmale von Bildungssprache, die die dargestellte Liste mit sprachlichen Merkmalen für das bildungssprachliche Register ergänzen würden (Biber \& Gray, 2013b; Celce-Murcia, 2002; Fang et al., 2006; Gogolin \& Lange, 2011; Schleppegrell, 2001, 2006).

Neben den zahlreichen möglichen Auflistungen von spezifischen sprachlichen Merkmalen des bildungssprachlichen Registers in einzelner Form gibt es weitere Tendenzen, die Merkmale in Beziehung miteinander zu setzen. Eine Möglichkeit bietet die Unterscheidung von grammatischen Formen, beispielsweise Lexik, Syntax, Morphologie sowie Wort-, Satz- und Textebene, wie in Anhang bei einigen Auflistung geschehen.

\subsubsection{Mathematische Fachsprache}

Wie bereits in Abschnitt 4.2.2 beschrieben, wird Fachsprache häufig in Kontrast zur Alltagssprache betrachtet. Unter Fachsprache können diejenigen Teile der Sprache verstanden werden, die in den zwischen den Gruppen einer Sprachgemeinschaft deutlich differenzierenden Lebensbereichen gebräuchlich sind und sich auf die dafür spezifischen Gegenstände, Sachverhalte und Vorstellungen beziehen (Kretzenbacher, 2008; Trabant, 1983).

Mathematisches Register: Auch im Bereich der mathematikdidaktischen Forschung ist, wie bereits erwähnt, das mathematische (fachsprachliche) Register von besonderer Relevanz. Im mathematikdidaktischen Diskurs verwendet Schweiger (1997) früh den Begriff des Registers für mathematisch-didaktische Inhalte. Der Begriff des numerischen Registers wird zur Beschreibung eines Teils der sprachlichen Äußerungen im Mathematikunterricht eingeführt. Im Vergleich zum mathematischen Register, das die sprachlichen Ressourcen, die benötigt werden, vollständig erfasst, betrachtet das numerische Register als Teil des mathematischen Registers nur einen Teil der sprachlichen Mittel. Seit der Nutzung des Registerbegriffs durch Schweiger (1997) ist der Begriff ein Standard in der Analyse und Diskussion von Sprache geworden.

Für das mathematische Register formuliert Halliday (1975) eine Definition durch eine funktionale Begründung der Verwendung von Sprache zur Bedeutungskonstruktion und der anschließenden Übertragung auf mathematische Inhalte aufgrund eines mathematischen Zwecks: 
[...] a set of meanings that is appropriate to a particular function of language, together with the words and structures which express these meanings. We can refer to a mathematics register, in the sense of the meanings that belong to the language of mathematics (the mathematical use of natural language, that is: not mathematics itself), and that a language must express if it is used for mathematical purposes (S. 65).

Das mathematische Register konzeptualisiert sich entsprechend der Verwendung der natürlichen Sprache zum Zweck der mathematischen Bedeutungskonstruktion. Halliday (1975) verweist dabei explizit auf die strikte Unterscheidung zwischen Sprache und Mathematik. Die Sprache bzw. die sprachlichen Merkmale, die genutzt werden, sind nicht die Mathematik selbst.

Sprachliche Merkmale des mathematischen Registers: Grundlegend scheint die Terminologie der Fachsprache ein besonderes Kennzeichen zu sein, doch es zeigt sich, dass die terminologische Distinktion zwischen Fachsprache und anderen Registern ihre Grenzen hat (Hoffmann, 2008; Rincke, 2010). Aus diesen und anderen Gründen plädiert Kalverkämper (1990), unter Einbezug des Vergleichs zwischen Alltagsprachlichkeit und Fachsprachlichkeit, für eine integrierte Betrachtung von Fach- und Alltagssprache. Die Beziehung zwischen Fachsprache und Alltagssprache wird in Abb. 4.1 dargestellt.

Zwischen der Kontext- (I) und Sprachebene (II) befindet sich das alltägliche Phänomen im Gegensatz zum fachlichen Phänomen. Besonders gekennzeichnet ist die Fachsprache durch einen hohen Merkmalsreichtum. Außerdem wird Fachsprache meist als präziser Sprachgebrauch verstanden, der gegenstandsspezifisch ist. Dagegen unterscheidet sich die Alltagssprache deutlich und kann als ungenau, inakkurat und diffus angesehen werden (Trabant, 1983). Im Kontext u. a. von Aussagen von Merkmalsreichtum und -armut ist diese Unterscheidung nicht hinreichend, um das mathematische (fachsprachliche) Register zu beschreiben (Jakob, 2008; von Hahn, 2008).

Nach Rincke (2010) muss die Fachsprache als referenzieller Begriff verstanden werden, der stets im Zusammenhang mit der assoziierten Domäne betrachtet werden muss. So wäre die mathematische Fachsprache die Sprache, die Mathematikerinnen und Mathematiker nutzen, um über Inhalte der Mathematik zu kommunizieren. Außerdem ergeben sich aufgrund der Spezifika der Fachsprache zusammenhängende Kommunikationsanforderungen und -situationen.

Neben der erläuterten Betrachtung von Fachsprache analysiert Rincke (2010) zentrale Dimensionen, die Fachsprache kennzeichnen. So lassen sich das für die Domäne spezifisch genutzte Vokabular, die Abgrenzung zwischen Fachsprache und Alltagssprache unter dem dominanten Aspekt des Wortschatzes sowie den 


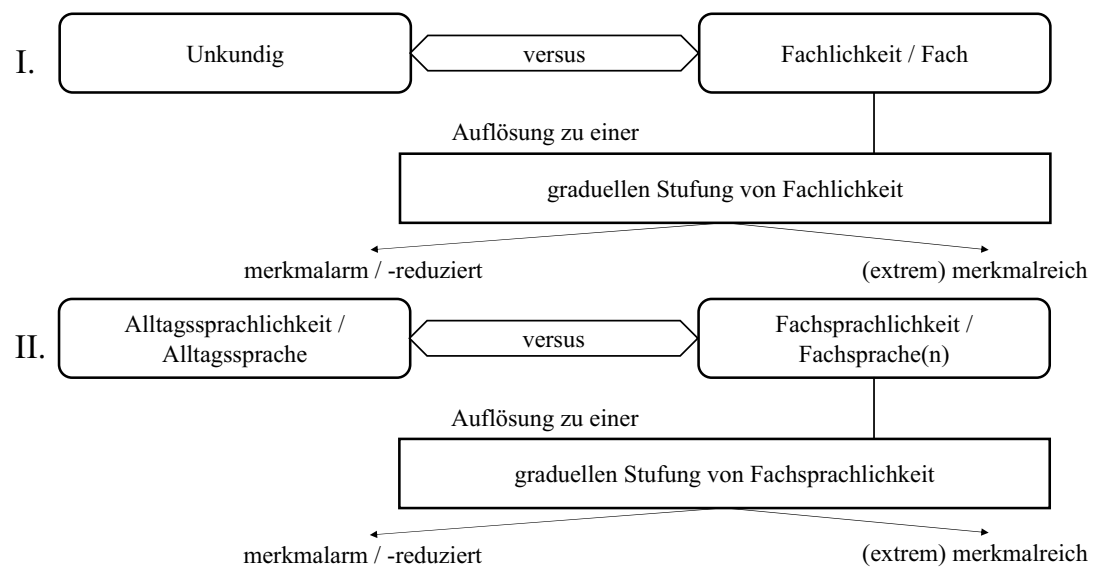

Abbildung 4.1 Skalen der Fachsprachlichkeit und Fachlichkeit nach Kalverkämper (1990, S. 123)

syntaktischen Mitteln, um Gegenstände zu beschreiben, die Ausbildung von speziellen Fachtextsorten und die Text-Kontext-Beziehungen, die für die Fachsprache eine Rolle spielen, unterscheiden (Halliday, 1993, 2004b; Lemke, 2012; Martin, 1993; Schleppegrell, 2006).

Eine Ergänzung zur Beschreibung des mathematischen (fachsprachlichen) Registers ist die Möglichkeit, die Fachsprache mit der Wissenschaftssprache in Beziehung zu setzen (Czicza \& Hennig, 2011; Trabant, 1983). Kretzenbacher (2008) verweist darauf, dass es zum Teil problematisch sein kann, die Wissenschaftssprache als Teilmenge der Fachsprache zu betrachten. Dahingehend ergeben sich drei Problemfelder, die im Folgenden genannt und diskutiert werden sollen: zum einen der Fokus der Fachsprache, der tendenziell auf der lexikalischen Ebene und der Verbindung mit der Alltagssprache liegt, zum Zweiten eine weitreichendere Bedeutung der Sprache für die Wissenschaft (als Erkenntnisapparat) im Gegensatz zum Fach (z. B. zu denen auch Bereiche gezählt werden können, die nicht auf Erkenntnisgewinn abzielen) und drittens eine klarere Definierbarkeit des Begriffs Wissenschaft im Vergleich zum Fach (Morek \& Heller, 2012).

Die drei genannten Problemfelder lassen sich im Kontext des mathematischen Registers wie folgt deuten. Das erste Problemfeld, die Betrachtung von Fachund Alltagssprache und die Fokussierung auf lexikalische Merkmale, hat für den 
Mathematikunterricht eine limitierende Beschreibungsfunktion, durch die erweiterte Betrachtung der Wissenschaftssprache können neben lexikalischen Merkmalen weitere für den Lehr-Lern-Prozess relevante Merkmale in Betracht gezogen werden (vgl. Abschnitt 4.2.4). Zum zweiten Problemfeld stellt sich dar, dass sich der Mathematikunterricht zwangsläufig auf die wissenschaftliche Bezugsdisziplin bezieht; insoweit scheint eine erweiterte Betrachtung durch die Wissenschaftssprache legitim. Das dritte Problemfeld bezieht sich auf die Definierbarkeit des Begriffs Wissenschaft, die sich schwieriger darstellt als für die Fachsprache, da hier die Alltagssprache nicht als Gegensatz dient. Die Schwierigkeit lässt sich nicht auflösen, jedoch können durch den Einbezug der Wissenschaftssprache weitere sprachliche Merkmale für das mathematische Register betrachtet werden, die als bedeutsam erachtet werden können. Um den Einbezug der Wissenschaftssprache durch eine Notation zu unterlegen, wird nachfolgend von mathematischer Fachsprache bzw. mathematischen fachsprachlichen Registern gesprochen.

Durch die von Czicza und Hennig (2011, S. 39) geleistete pragmatische Analyseebene ergibt sich eine Ergänzung zur Klärung der Deutung der Aspekte der Wissenschaftssprache, die sich ebenfalls für die mathematische Fachsprache als relevant herausstellen. Das in Abb. 4.2. dargestellte Modell stellt zentrale Unterscheidungskriterien der Verwendung von Wissenschaftssprache dar. Das Axiom der Wissenschaftssprache wird unter dem Aspekt der Erkenntniszuwachsorientierung betrachtet. Diese lässt sich als höchste Ebene auch für die mathematische Fachsprache abbilden und zeigt damit die enge Verbindung zwischen mathematischer Fachsprache und Wissenschaftssprache. Aus dem Axiom leiten sich unter den Punkt Gebote vier Aspekte ab.

Die Ökonomie als Maßgabe um Redundanz in Texten zu reduzieren, und damit unnötige Informationen, die nicht im Erkenntnisinteresse des Gegenstands sind, aus dem Text zu entfernen. Die Kondensation des Textes findet mit sprachlichen Mittel wie Komposita statt.

Präzision dient zur Vermeidung von Doppeldeutigkeiten und Unschärfe; der Erkenntnisgegenstand sollte klar und deutlich ausgedrückt werden, um deutlich darzustellen, was gemeint ist.

Origo-Exklusivität (Anonymität) ist die Zielsetzung wissenschaftlicher Kommunikation; der Erkenntnisgegenstand soll möglichst ohne überdeutliche interpersonelle Referenzen dargestellt werden und lässt sich als sprachlicher Ausdruck der Objektivität deuten. Realisiert wird die Objektivität durch Verallgemeinerungen im Bereich der erwähnten interpersonellen sprachlichen Formen durch Passiv und die Verwendung der dritten Person, aber auch durch spezifische Strukturierung der Zeit im Präsens. 


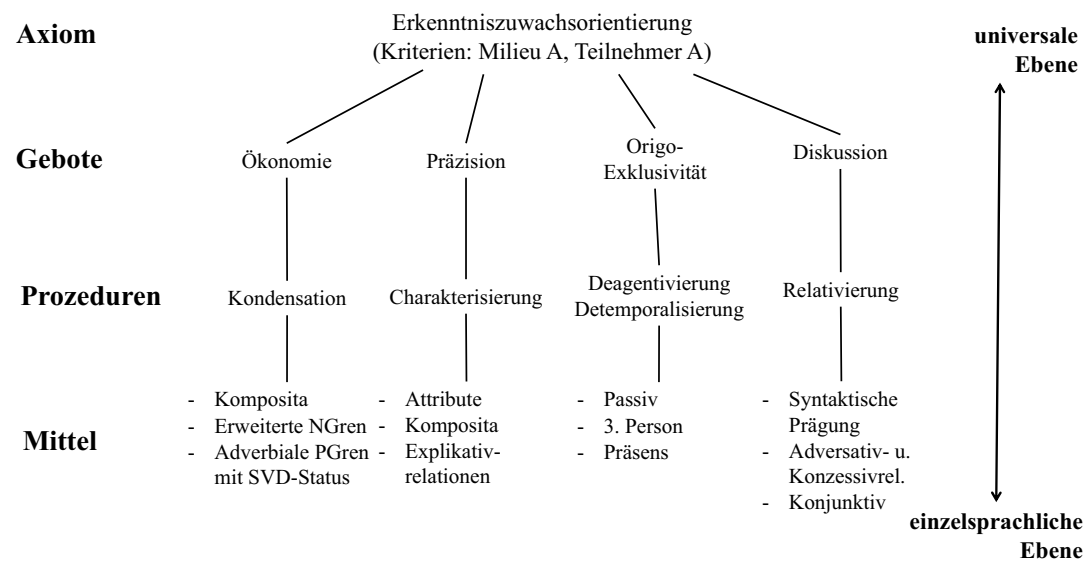

Abbildung 4.2 Modell der Wissenschaftskommunikation A: Pol maximaler Wissenschaftlichkeit nach Czicza und Hennig (2011, S. 50)

Diskussion ist die Voraussetzung für Erkenntnisgewinn und damit ebenfalls ein Kernelement wissenschaftlicher Kommunikation. Ohne den sozialen Austausch findet keine Erkenntnis statt. Dabei ist vor allem die Diskutierbarkeit ein bedeutender Aspekt der wissenschaftlichen Kommunikation. Daraus entwickeln sich Relativierung und daher Wahrscheinlichkeitsaussagen, Unbestimmtheit und Möglichkeiten, die sich u. a. in Modalformen und Konjunktiven ausdrücken.

In Abb. 4.3 ist das zweite Modell abgebildet, das ebenfalls die Alltagssprache zur Kontrastierung der Charakterisierung der Wissenschaftssprache nutzt. In diesem Modell ist die kommunikative Orientierung für die vier wissenschaftssprachlichen Dimensionen abgebildet. Die Wissenschaftssprache bzw. mathematische Fachsprache hat ein expansives Verhalten in Bezug auf den Erkenntnisgewinn, gegenüber zeigt sich für die Alltagssprache tendenziell kein Bezug zu einem Erkenntnisgewinn.

Der Einbezug der Wissenschaftssprache ermöglicht eine erweiterte Betrachtung von sprachlichen Merkmalen, die für das mathematisch-fachsprachliche Register als bedeutsam erachtet werden können. Besonders die von Abb. 4.2 und Abb. 4.3 dargestellten Modelle zeigen deutlich die Verbindung von wissenschaftssprachlichen Aspekten und der mathematischen Fachsprache, was deutlicher wird, wenn in Betracht gezogen wird, dass die in den Abbildungen dargestellten sprachlichen Merkmale als potenzielle sprachliche Hürden für Textaufgaben gelten (Prediger, 2013a). 
Wissenschaftssprache / Alltagssprache

Mathematische Fachsprache

(keine Erkenntniszuwachsorientierung)

(Erkenntniszuwachsorientierung)

mehr

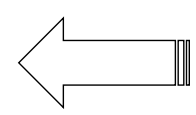

mehr
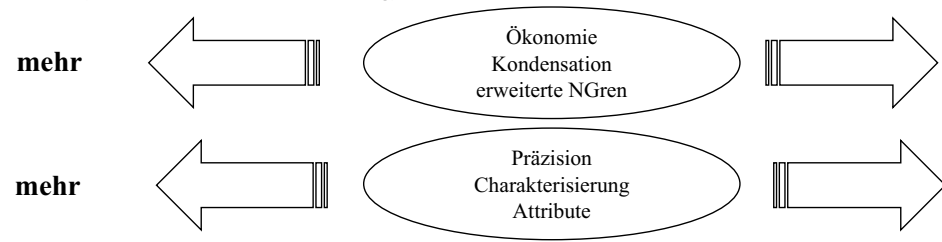

weniger
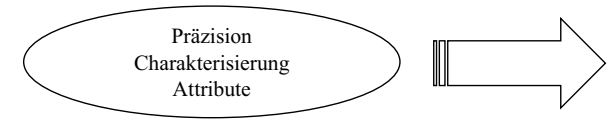

weniger

mehr
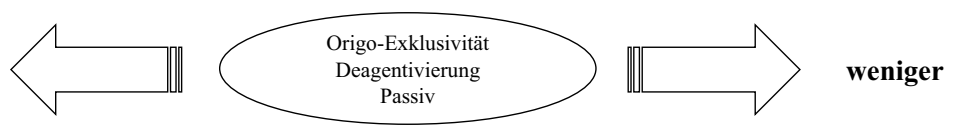

mehr
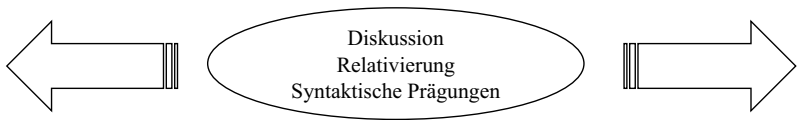

weniger

Abbildung 4.3 Modell der Wissenschaftskommunikation B: reduktives und expansives Verhalten von Wissenschafts- und Alltagssprache nach Czicza und Hennig (2011, S. 55)

Resümee (Abschnitt 4.2): Sprachliche Variationen bilden die Flexibilität und den Wandel von Sprache in der Nutzung wieder. In der Unterscheidung von Dialekt und Register können die häufigsten Phänomene von sprachlicher Variation differenziert werden. Das Konzept des Registers ist für Lehr-Lern-Prozesse in der Schule bedeutsam, da das Register sprachliche Variationen betrachtet, die mit situativen Parametern in Verbindung stehen. Dies betrifft u. a. die Institution Schule. Der Begriff Register kann in unterschiedlicher Weise interpretiert werden, grundlegend sind besonders Wahrscheinlichkeitsaussagen über sprachliche Merkmale, die in bestimmten Situationen frequentiert vorkommen. Für den Mathematikunterricht sind alltagssprachliche, schulsprachliche, bildungssprachliche und mathematisch-fachsprachliche Register interessant, die jeweils unterschiedliche sprachliche Merkmale nutzen und notwendig sind, um die fachlichen Inhalte zu vermitteln.

\subsection{Variationen von Registern}

Das Konzept des Registers klassifiziert spezifische Sprachhandlungsprozesse aufgrund der häufigen Nutzung von einzelnen sprachlichen Ressourcen. Damit 
erscheinen diese Gruppen von Registern in der theoretischen Formulierung diskret. Die Modellannahme von scheinbar klar abgrenzbaren Gruppen von Registern kann jedoch nicht beschreiben, wie sich die einzelnen Register in der Nutzung abbilden. Wenn die Register als sprachliche Ressourcen erworben sind, werden sie in der Sprachhandlung kontinuierlich genutzt und sie variieren durch die Verwendung in den jeweiligen Situationen. Die Variation von Registern in der Nutzung beschreibt die Schwierigkeit, Register empirisch zu determinieren.

Die Verwendung von Sprache hat einen Einfluss auf die Variabilität der genutzten sprachlichen Merkmale. Damit ist ebenfalls von einer Variabilität der Register auszugehen, da eine bedeutende Eigenschaft von Registern ist, dass sie die Verknüpfung und die Kombination von lexikalischen und grammatischen Eigenschaften von Sprache bzw. Text konstituieren (Ure \& Ellis, 2014). Die Variabilität der Verwendung von Registern lässt sich empirisch ableiten und wird durch Registeranalysen betrachtet. Ure und Ellis (2014) bezeichnen den Untersuchungsgegenstand von Registeranalysen als die Analyse von sprachlichen Handlungen in Ausprägung von Text (und Diskurs) und des Kontextes der Situation. Register können untersucht werden, indem eine hohe Anzahl an Sprachhandlungen, beispielsweise Texte, betrachtet und daraus generalisierte Schlüsse über sprachliche Merkmale gezogen werden, die diese Texte teilen. Dahingehend verweisen Ure und Ellis (2014) darauf, dass ein bestimmtes Register, beispielsweise das bildungssprachliche Register, in einer idealen, diskreten, abgrenzbaren und definierbaren Form nicht existiert, dass dieser Text je nach Eigenschaft und Länge nur eine gewisse Anzahl an Registermerkmalen besitzt und dass sich die Einzelbetrachtung von Fällen als irreführend darstellen kann:

the ideal example of text in a specific register exists no more than does the ideal man in the street. Moreover, register is a property of the textual part of a language event which may be short or may be very long. The longer the text, the more register features it will carry, but we cannot demand that all these register features be packed into a short example, particularly not into a single-sentence example. Many register features take the form of inter-sentence linguistic relations: grammatical and lexicogrammatical cohesion [...] and lexical collocations [...] Single sentence examples can give a misleading picture of what register really is (S. 205).

Die Unschärfe, die bei der Betrachtung von Registern in der tatsächlichen sprachlichen Verwendung entsteht, impliziert die Variationen von Registern (Registervariationen) (Biber, 2006). Dass Registervariationen verbreitet sind, beschreibt Ure (1982) damit, dass jede Sprachgemeinschaft ein eigenes Registersystem bildet und dieses entsprechend dem Tätigkeitsbereich, in dem die Mitglieder agieren, anpasst und flexibel verwendet. Ein solches Registersystem wird für den 
Mathematikunterricht in den diskutierten typischen Registern in den Abschnitten 4.2.2 (alltagssprachliches Register) bis 4.2.5 (mathematisches fachsprachliches Register) realisiert.

Ferguson (1983) bezeichnet Registervariationen, bei denen sich die Sprachstrukturen je Verwendungszweck verändern, als allgegenwärtig in der Sprache. Dadurch, dass kein Mensch durchgängig auf die gleiche Weise spricht, kann eine Vielzahl von Registern angetroffen werden. In Anbetracht des Mathematikunterrichts wird ersichtlich, dass das Registersystem flexibel angepasst und verwendet wird und nicht nur die sprachlichen Merkmale eines Registers in einer statistischen Sichtweise Verwendung finden. So stellt sich für den Mathematikunterricht eine variantenreiche Nutzung der in Abschnitt 4.2.2 bis 4.2 .5 beschriebenen Register dar. Dahingehend spricht eine Lehrkraft im Mathematikunterricht nicht nur bildungssprachlich und verwendet registertypische sprachliche Merkmale, sondern variiert ihre Sprache und wechselt innerhalb einer Unterrichtsstunde je nach situativen Voraussetzungen zwischen den verschiedenen Registern.

Beschrieben werden können Registervariationen als sprachliche Unterschiede, die mit der Änderung der Situationen korrelieren (Biber, 2006; Ferguson, 1994). Finegan und Biber (2001) definieren Registervariationen als Variation der Häufigkeit von sprachlichen Merkmalen. In ähnlicher Weise betrachtet Halliday (2005) Registervariationen, indem er die Frequenz von sprachlichen Merkmalen als zentralen Gegenstand von Registervariationen bezeichnet (Biber \& Reppen, 2002). So zeigt Svartvik (1985), dass die Frequenz von sprachlichen Merkmalen von Registern in einer Sprache variieren kann.

Zur Analyse von Registervariationen wird theoretisch angenommen, dass die sprachlichen Merkmale spezifische sprachliche Funktionen erfüllen, die nicht allein durch Konventionen erklärbar sind (Finegan \& Biber, 2001). Beispielsweise lassen sich für E-Mails charakteristische sprachliche Merkmale identifizieren, zum Beispiel zu Beginn die Begrüßung Hallo Philipp, Sehr geehrte Damen und Herren etc. und abschließend Viele Grüße. Diese sprachlichen Merkmale sind konventionsbezogene Merkmale und aufgrund ihrer kommunikativen Funktion nicht funktional (Finegan \& Biber, 2001). Nach Finegan und Biber (2001) können sich sprachliche Merkmale auf funktionaler Basis deutlich unterscheiden. So sind Texte mit häufigen Substantiven deutlich von Texten mit vielen Pronomen zu unterscheiden, nämlich dadurch, dass die Texte mit Pronomen kontextualisiert sind, im Gegensatz zu Texten mit vielen Substantiven. Die Frequenz für Pronomen in kontextbezogenen Texten ist so deutlich, dass Finegan und Biber (2001) Pronomen als sprachliche Marker zur Feststellung von alltagssprachlichen Texten nutzen. Das häufige Vorkommen von Pronomen in alltagssprachlichen Texten 
basiert nicht auf konventionellen Bedingungen, sondern ergibt sich aus funktionalen Gründen, was dazu führt, dass Pronomen in kontextualisierten Situationen häufiger gewählt werden.

Biber (2006) erörtert Registervariationen insbesondere als ein empirisches Phänomen. Dahingehend ergeben sich unterschiedliche Analysemöglichkeiten von Registervariationen. Diese Analysemöglichkeiten können in vier Kategorien unterschieden werden: synchrone Beschreibungen eines einzelnen Registers, diachrone Beschreibungen, die die Entwicklung eines einzelnen Registers untersuchen, synchrone Beschreibungen der Variationsmuster zwischen mehreren Registern und diachrone Beschreibungen, die Änderungen in den Variationsmustern zwischen mehreren Registern betrachten. Die Analysen fokussieren den Vergleich von Variationsdimensionen zwischen Sprachen oder zwischen der zeitbedingten Veränderung einer Sprache. Damit ist die Zielsetzung darauf ausgelegt, gemeinsame oder unterschiedliche Muster der Registervariationen zu bestimmen.

Vor dem Hintergrund der in Abschnitt 2.4.3 beschriebenen Problematik, dass nicht alle Lernenden Zugang zu den gleichen sprachlichen Ressourcen besitzen und entsprechend nicht gleich kompetent bei der Nutzung unterschiedlicher Register sind, ist eine Analyse der Registervariationen unter der Perspektive sprachlicher Hürden im Mathematikunterricht zur Bestimmung der sprachlichen Bedingungen, die vorausgesetzt werden, notwendig. Dahingehend sind für die Entwicklung eines Instruments zur sprachlichen Variation von Textaufgaben Registervariationen zu beachten. Im folgenden Abschnitt 4.4 werden methodische Verfahren beschrieben, die die Analyse von Registervariationen beschreiben.

\subsection{Korpusbasierte Ansätze zur Analyse von Registervariationen}

Variationen von Registern treten auf, wenn empirisch geprüft wird, welche sprachlichen Merkmale in wechselnden situativen Voraussetzungen vorkommen. Wie in den Abschnitten 3.3.4 und 4.3 ist eine Einzelfallbetrachtung von Texten nicht ausreichend, um Register zu untersuchen. Es wird eine Vielzahl von Fällen benötigt, die das Sprachsystem in ausreichendem Maße repräsentiert. Eine Möglichkeit zur Analyse von Registervariationen bieten aufgrund dieser Prämisse korpusbasierte Ansätze.

Ein Korpus kann in sprachanalytischem Sinn als eine Kollektion von Texten verstanden werden, die auf natürliche Weise vorkommen. Diese Textkollektion wird gewählt, um einen bestimmten Zustand oder eine Varietät von zu charakterisierender Sprache festzustellen (Balossi, 2014; Sinclair, 1991; Viana et al., 
2011). Die Zielsetzung des korpusbasierten Vorgehens sind die Beobachtung, die Beschreibung und die Interpretation von stilistischen Merkmalen der Sprache in literarischen und nichtliterarischen Texten (Balossi, 2014; Zyngier et al., 2008). Für die Verwendung des Ansatzes spricht die Möglichkeit der Nutzung von Software und von bestehenden großen Korpora-Kollektionen, beispielsweise der Leipzig Corpora Collection, die die Analyse mit einem Referenzkorpus erleichtern. Der Einsatz von computergestützten Methoden wird dabei in vielen unterschiedlichen Bereichen der sprachwissenschaftlichen Forschung verwendet. Balossi (2014) fasst für den Korpus-Ansatz drei charakteristische Merkmale zusammen:

1. Empirisch: Die korpusbasierte Analyse ist empirisch, indem die tatsächlichen Muster von natürlichen Texten als Grundlage der Untersuchung verwendet werden.

2. Technisiert: Korpora werden in Form von computerbasierter Analyse in digitalisierten Modi konstruiert und sind so zugänglich für weitere Analysen.

3. Methodisch: In der weiteren Verwendung des digitalisierten Korpus können für weitere Analysen sowohl quantitative als auch qualitative Analyseverfahren verwendet werden.

Diese drei Merkmale werden in der praktischen Nutzung der Analyse von Korpora unterschiedlich umgesetzt und verwendet.

Die Technisierung in Form von computerbasierter Analyse hat insbesondere den Fokus, natürliche Texte für computerbasierte Verfahren zur Verfügung zu stellen. Es wird grundlegend unterschieden zwischen nicht annotierten Texten, das heißt, Texten ohne Markierung, beispielsweise rohe bzw. reine Texte in Form von Absätzen, Sätzen und Satzzeichen, und annotierten Texte, also Texten, die eine spezifische Markierung erhalten. Die Annotation bezeichnet die Klassifizierung von Texten durch die sprachlichen Bestandteile (Archer, 2007; Balossi, 2014; McEnery \& Hardie, 2012). Die Korpusannotation kann dabei mittels Computerprogramm automatisch oder durch Experten oder Probanden manuell erfolgen. In der Praxis werden manuelle und computerbasierte Annotationen kombiniert (Balossi, 2014; Semino \& Short, 2004). Die automatische Annotation von Texten wird dabei als Tag bzw. Tagging bezeichnet. Tagging leitet sich von der Bezeichnung für die Markierung von Wörtern in einem Korpus durch Etikettierung (Tag) ab. Durch das Tagging ist es möglich, zusätzliche Informationen über grammatikalische, semantische und pragmatische Funktionen oder den sprachlichen Kontext zu erhalten (McEnery \& Hardie, 2012). 
Für die Markierung von Texten werden unterschiedliche Formen von Annotationen unterschieden (Balossi, 2014; Leech, 2005). Eine der häufigsten Formen der Markierung ist die Part-of-speech(POS)-Annotation, auch POS-Tagging. Beim POS-Tagging wird den Elementen des Korpus eine allgemeine Wortklasse zugewiesen, beispielsweise wird das Wort Winkelhalbierende mit der Wortklasse Substantive markiert. Weitere Möglichkeiten sind semantische Annotationen, die durch die Bildung von semantischen Kategorien charakterisiert sind. So unterscheidet die semantische Annotation zwischen dem Begriff Wurzel auf der Ebene der Botanik und der Algebra. Daneben existiert das lexikalische Tagging, bei dem Wortformen des gleichen Lexems abgeleitet werden, beispielsweise werden für das Lexem addieren die zugehörigen Wortformen addiert, addiere und addierte angezeigt.

Neben diesen als klassisch zu betrachtenden Annotationsformen existieren Markierungen auf höherer sprachlicher Ebene. Eine Form ist die pragmatische Annotation. Diese zeichnet sich durch das Hinzufügen von Informationen aus. So kann die Phrase [...] parallele Geraden konstruieren [...] als Imperativ begriffen („Du sollst eine parallele Gerade konstruieren!“) oder als Frage interpretiert werden (,Kannst du parallele Geraden konstruieren?“). Das Hinzufügen der pragmatischen Instanz zeichnet diese Form des Tagging aus.

Neben der pragmatischen Annotation ist die linguistische Annotation eine Klassifikation auf einer höheren Ebene von Sprachphänomenen; so wird damit beispielsweise direkte und indirekte Sprache klassifiziert. Der Vorteil, der sich aus der Klassifikation sprachlicher Merkmale ergibt, ist das Hinzufügen weiterer interpretativer sprachlicher Informationen.

Ein besonderer Vorteil der korpusbasierten Analyse ist der einfache Transfer für weitere methodische Analysen. Es können dabei sowohl qualitative als auch quantitative Methoden verwendet werden. Für qualitative Methoden wird das Tagging als Basis zur Identifizierung und Beschreibung von Sprache genutzt (Biber et al., 1998, 2002, 2016). Für quantitative Methoden ergibt sich durch das automatische Tagging die Möglichkeit, zum einen die Häufigkeiten von Wörtern in einem Korpus darzustellen (vgl. Abschnitt 4.5.1) und zum anderen weitere nichtdeskriptive statistische Modelle zu nutzen (vgl. Abschnitt 4.5.3). Bezüglich quantitativer Methoden konstatiert Biber (2006), dass sie häufig nur deskriptiv verwendet werden. Er plädiert für korpusbasierte Analysen, die über die einfache Bestimmung der Anzahl von sprachlichen Merkmalen hinausgehen. Dahingehend werden in Abschnitt 4.5 unterschiedliche Möglichkeiten der quantitativen Analyse von Korpora dargestellt. 


\subsection{Methoden zur Analyse von Registervariationen}

Korpusbasierte Verfahren ermöglichen es, eine Vielzahl von Texten zu analysieren. Damit wird grundlegend die Analyse von Registern und Registervariationen ermöglicht, die nur durch eine Stichprobe von vielen Fällen (Korpus) möglich ist. Ein Korpus soll die Varianz der verwendeten sprachlichen Mittel abbilden, um so Ähnlichkeiten und Unterschiedlichkeiten festzustellen.

Überblick (Abschnitt 4.5): In der Analyse eines Korpus kann die Häufigkeit von einzelnen sprachlichen Merkmalen untersucht werden (Abschnitt 4.5.1). Außerdem kann das gemeinsame Vorkommen von sprachlichen Merkmalen betrachtet werden, also die Identifikation von sprachlichen Merkmalen, die empirisch häufig in Texten vorkommen (Abschnitt 4.5.2). Zur Analyse des gemeinsamen Vorkommens von sprachlichen Merkmalen werden multivariate Verfahren genutzt, um die sprachlichen Muster zu klassifizieren (Abschnitt 4.5.3).

\subsubsection{Betrachtung von einzelnen sprachlichen Merkmalen}

Registeranalysen, die nur einzelne sprachliche Merkmale untersuchen, betrachten die Häufigkeiten, die sich ableiten lassen, durch unterschiedliche Verwendungszwecke dieser Merkmale. Nach Conrad (2015) dient eine solche Analyse nicht dazu, Register selbst zu beschreiben, jedoch kann durch ein solches Vorgehen eine Vielzahl von Informationen hergestellt werden, über die sprachliche Merkmale in den verschiedenen Registern dargestellt werden. Die Analyse von einzelnen sprachlichen Merkmalen wurde von unterschiedlichen Studien verwendet. Conrad (2015) liefert eine Auswahl an Studien, die sich am Begriff der Registervariationen orientierten. In diesen Studien wird durch Häufigkeitsanalysen beispielsweise das Vorkommen von Adjektiven, Nominalisierungen, Passiv oder lexikalischen oder grammatikalischen Merkmalen bestimmt und verglichen.

Neben Studien, die den Bezug zu Registeranalysen und Analysen zu Registervariationen herstellten, existieren weitere Studien, die nicht speziell darauf verweisen, jedoch ebenfalls den Ansatz der Analyse von einzelnen sprachlichen Merkmalen verfolgen, die mit einem Register in Beziehung gesetzt werden (Haag et al., 2015; E. Johnson \& Monroe, 2004). 


\subsubsection{Gemeinsames Vorkommen von sprachlichen Merkmalen}

Einige sprachliche Merkmale treten in gehäufter Weise gemeinsam mit anderen Merkmalen auf. Das gemeinsame Auftreten von sprachlichen Merkmalen wird in der Literatur unterschiedlich aufgegriffen, je nach Analysegegenstand entweder theoretisch oder empirisch und häufig in Hinblick auf die Textfunktion beschrieben (Eggs, 2008; Feilke, 2012b; Gogolin et al., 2007; Gülich \& Hausendorf, 2008; Heinemann, 2008; Hoffmann, 2008; Jahr, 2008; Rolf, 2008).

Biber (2006) begründet das gemeinsame Vorkommen von sprachlichen Merkmalen, wie in Abschnitt 4.2 geschildert, funktional. Dies bedeutet, dass das gemeinsame Vorkommen nicht durch Konventionen erklärbar ist, wie beispielsweise in der Formulierung einer E-Mail.

Feilke (2012b) konzipiert in Bezugnahme zum bildungssprachlichen Register eine funktionale Gruppierung von sprachlichen Merkmalen. Den einzelnen sprachlichen Merkmalen werden in diesem Modell grundlegend zwei Äußerungsaspekte zugeordnet, zum einen der Inhaltsaspekt und zum anderen der Beziehungsaspekt. Der Inhaltsaspekt und der Beziehungsaspekt werden in zwei Sprecherstrategien untergegliedert. Der Inhaltsaspekt, in dem die Aussageinformationen im Vordergrund stehen, wird differenziert in das Explizieren und das Verdichten. Der Beziehungsaspekt bezieht sich auf die Sprecherabsicht, die die Strategien des Verallgemeinerns und Diskutierens zusammenfasst.

Im Bezug zum Inhaltsaspekt werden dem Explizieren „komplexe Adverbiale, Attribute und Sätze zugeordnet und dem Verdichten Nominalisierung, Komposita, Partizipialattribute (die siebende Flüssigkeit), Präpositionaladverbien (unter Druck, durch Erhitzen)“ zugeordnet (Feilke, 2012b, S. 8).

Im Beziehungsaspekt werden für das Verallgemeinern sprachliche Mittel zusammengefasst wie: „verallgemeinernden (generischen) Formen, z. B. Verwendung der 3. Person, Vermeidung der 1. und 2. Person, Ausblendung des Handlungsträgers (Deagentivierung, z. B. Passiv-, man-, lassen-Konstruktionen, z. B. es wird gezeigt, dass; man kann zeigen, dass; es lässt sich zeigen, dass; kommt es dazu, dass)“. Für das Diskutieren werden sprachliche Mittel wie „Modalverben, Modalisierungen, z. B. Konjunktivformen (würde bedeuten, dass, hätte zur Folge)“" zusammen betrachtet (Feilke, 2012b, S. 8).

Wird die Unterscheidung der Äußerungsaspekte mit den in Abschnitt 2.3 erläuterten sprachlichen Funktionen betrachtet, ist die Verknüpfung von sprachlichen Funktionen und den erläuterten Aspekten legitim und begründet die funktionale Einteilung der sprachlichen Merkmale. Besonders für eine funktionale Verknüpfung eignen sich die in Abschnitt 2.3.3 geschilderten Metafunktionen. Die ideelle 
Metafunktion lässt sich mit dem Inhaltsaspekt und die interpersonale Metafunktion mit dem Beziehungsaspekt in Beziehung setzen. Ergänzend zu dieser funktionalen Zuordnung wird in Abschnitt 6.4 die Gruppierung um den Textaspekt in Verbindung mit der textuellen Metafunktion ergänzt.

Neben der erläuterten Gruppierung von Textmerkmalen lassen sich für die Verwendung von Sprache, unterschiedliche Strategien der Vertextung unterscheiden, die sich durch bestimmte Textorganisationen und Textmerkmale auszeichnen. Gemäß Werlich (1976) lassen sich fünf unterschiedliche Strategien der Vertextung (Text-Types) differenzieren. Die erste Form ist das Vertextungsmuster der Deskription. Die Deskription zeichnet sich durch das Darstellen von Objekten durch die Vermittlung von konkreten Merkmalen, Kennzeichen und Besonderheiten aus (Heinemann, 2008). Die zweite Form ist das Vertextungsmuster der Explikation. Die Explikation dient der Erläuterung von Handlungen, Behauptungen, Wünschen etc. (Jahr, 2008). Die dritte Form ist das Vertextungsmuster der Narration. Narrative Texte werden genutzt, um zeitliche und geschichtliche Prozesse darzustellen (Güllich, 2008). Eine weitere Vertextungsform ist die der Argumentation. Argumentative Texte orientieren sich teilweise an Aspekten der Explikation, mit der gewisse Konzepte, Ideen oder Annahmen dargestellt werden. Die Besonderheit stellt das Ziel dar, Gründe für oder gegen einen Inhalt zu präsentieren (Eggs, 2008). Die fünfte Form ist das instruktive Vertextungsmuster. Instruktive Texte werden genutzt, um den Lesenden zu verdeutlichen, was verlangt wird und zu tun ist, mit einer direkten Zweckbindung des Texts auf die Handlung (Werlich, 1976). Die fünf Formen von Vertextung zeichnen sich durch die Verwendung von spezifischen Textmarkern aus. Diese können sich auf die Verwendung von Verben und Adverben oder die Nutzung von gewissen Substantiven beziehen (Werlich, 1976). Aufgrund des direkten Bezugs zwischen Vertextungsstrategien und Textmerkmalen können die unterschiedlichen Vertextungsstrategien dazu dienen, das gemeinsame Vorkommen von Textmerkmalen zu deuten. Aus diesem Grund werden in Abschnitt 7.2.5 die empirisch ermittelten, zusammen vorkommenden Textmerkmale auf gewisse Vertextungsstrategien zurückbezogen, um geeignete tragfähige Bezeichnungen für die empirischen Ergebnisse (Faktoren) zu erhalten.

\subsubsection{Multivariate Verfahren zur Analyse des gemeinsamen Vorkommens von sprachlichen Merkmalen}

Wenn für die Analyse von Registervariationen das gleichzeitige Auftreten von sprachlichen Merkmalen untersucht wird, muss das Ziel der Untersuchung sein, 
die Varianz im Auftreten unterschiedlicher sprachlicher Merkmale von Registern $\mathrm{zu}$ untersuchen.

Biber (2006) verwendete zur Analyse des gemeinsamen Vorkommens das multivariate Verfahren der Faktorenanalyse, die er aufgrund der Bildung von Dimensionen als multidimensionales Verfahren zur Analyse von Sprache respektive der sprachlichen Merkmale bezeichnet. Für das multivariate Verfahren wird angenommen, dass sprachliche Merkmale, die durch das gehäufte gemeinsame Auftreten eine Gruppe bilden, eine gemeinsame sprachliche Funktion miteinander teilen (Biber et al., 1998, 2016; Biber \& Conrad, 2019; Biber \& Gray, 2013a; Conrad, 2015).

Wie erwähnt, interpretiert Biber (2006) jeden Faktor der Faktorenanalyse als eine Dimension der Variation der sprachlichen Merkmale, da sich bei den Faktoren empirisch festgestellte Unterschiede ergeben. Durch die Faktorenanalyse werden die sprachlichen Merkmale, die in einem Datensatz analysiert werden sollen, systematisiert. Dadurch entstehen spezifisch textuelle Muster. Der multivariate Ansatz wird genutzt, um durch ein quantitatives Verfahren sprachliche Merkmale explorativ zu systematisieren (Biber \& Egbert, 2018; Conrad, 2015). Neben der Betrachtung der explorativen Faktorenanalyse von Biber (2006) als Mittel zur Registeranalyse sieht Pause (1984) die Faktorenanalyse und die Faktorenbewertung als mögliche Informationsquellen für die Analyse des Textverstehens. Die explorative Faktorenanalyse bzw. multivariate und strukturentdeckende Verfahren bieten daher reichhaltige Möglichkeiten der Analyse von Sprache im fachlichen (mathematischen) Kontext.

Das Vorgehen des Ansatzes kann in drei Stufen unterschieden werden: erstens die Bestimmung der Ausprägung der sprachlichen Merkmale je Aufgabe, zweitens die Verwendung der Faktorenanalyse, um die Faktoren der textuellen Muster festzustellen, drittens die funktionale Interpretation der textuellen Muster bei den Faktoren (Biber \& Egbert, 2018). Die Faktoren der Faktorenanalyse können sowohl auf sprachlicher Ebene als auch auf funktionaler Basis beschrieben werden. Die sprachliche Ebene ist durch die textuellen Muster der sprachlichen Merkmale bestimmt, die bei einem Faktor besonders repräsentiert werden. Auf funktionaler Basis können die Faktoren durch die funktionalen Merkmale interpretiert werden, die die sprachlichen Merkmale am häufigsten teilen. Für die funktionale Interpretation wird angenommen, dass die textuellen Muster zugrundeliegende Kommunikationsfunktionen widerspiegeln (Biber \& Egbert, 2018).

Gemäß Biber und Egbert (2018) werden auf Basis der Identifikation der Faktoren durch die Interpretation der spezifischen textuellen Muster je Faktor 
Bezeichnungen gewählt, die der Kommunikationsfunktion entsprechen. Die Herstellung und die Interpretation der textuellen Muster der Faktoren bieten die Möglichkeit, zu bestimmen, welche besonderen sprachlichen Voraussetzungen für die kommunikativen Funktionen dieser Texte erfüllt werden müssen. Für die Interpretation der Faktoren ist der Einbezug der Ähnlichkeiten und der Unterschiede der Faktoren zentral.

Der multivariate Ansatz vereinigt sowohl makroskopische Analysen, die die Beschreibung der typischen sprachlichen Merkmale von Registern betreffen, als auch mikroskopische Analysen, die die detaillierte Analyse sprachlicher Merkmale in bestimmten Texten anbelangen (Biber, 1985, 2006; Biber \& Gray, 2013a). Dies geht mit der Annahme einher, dass Verallgemeinerungen bezüglich Registervariationen auf einem breiten Spektrum an unterschiedlichen Registern basieren müssen und es nicht ausreicht, einzelne sprachliche Merkmale zu betrachten, um die Ähnlichkeiten oder Unterschiede von Registervariationen zu erfassen (Biber, 2006; Biber et al., 2002; Biber \& Reppen, 2002). Zur Analyse von Registervariationen nutzt Biber (2006) beispielsweise lexikalische Merkmale wie Type-token-Verhältnisse, Wortlänge; grammatische Merkmale wie Substantive, präpositionale Phrasen, Adjektive; und syntaktische Merkmale wie Relativsätze, Adverbialsätze.

Für den Vergleich der Faktoreninterpretation empfehlen Biber und Egbert (2018) die Bildung von Faktorenbewertungen anhand der Faktorenwerte. Zwar handelt es bei der Analyse von Registervariationen grundlegend um ein empirisches Verfahren, jedoch ist für die Interpretation dieser Muster ein qualitatives Vorgehen notwendig. Es findet dahingehend eine Synthese zwischen den quantitativen Ergebnissen und der qualitativen Interpretation der Variationsdimensionen statt. Die Interpretation geschieht insbesondere aufgrund der kommunikativen Funktion der einzelnen Merkmale. Für jede Variationsdimension wird in Hinblick auf die kommunikative Funktion ein Label als interpretative Bezeichnung vergeben, beispielsweise narrative vs. nichtnarrative Anliegen oder explizite vs. situationsabhängige Referenz (Biber, 2006).

Das multivariate Verfahren wird insbesondere als hochstatistische und in hohem Maß zeitaufwendige Analyse betrachtet (McEnery \& Hardie, 2012). Nach Conrad (2015) hat das multivariate Verfahren als Methode zur Betrachtung von Mustern von sprachlichen Merkmalen insbesondere in der empirischen Bildungsforschung noch keine große Verbreitung gefunden. Gründe können die bisher geringe Integration von multivariaten Verfahren mit qualitativen Techniken zur Analyse von Sprache und die ökonomische Perspektive sein. 


\subsection{Zusammenfassung}

Die Verwendung von sprachlichen Mitteln ist durch ständige Veränderungen geprägt, die sich während der Nutzung ergeben. Ein Instrument, das die Sprache bei Textaufgaben im Mathematikunterricht verändern soll, muss sich zwangsläufig an den typischen Variationen orientieren, die sich durch die Nutzung von Sprache im Mathematikunterricht ergeben.

Sprachliche Variationen sind Teil der menschlichen Sprache und können unterschieden werden in Dialekt und Register. Register beziehen sich auf Variationen, die mit situativen Veränderungen in Beziehung stehen, und sind damit von besonderer Bedeutung für die Betrachtung für die Schule und den Mathematikunterricht.

Register sind durch die gehäufte Verwendung von sprachlichen Merkmalen in gewissen Situationen ausgezeichnet. Für den Mathematikunterricht lassen sich typischerweise das alltagssprachliche, schulsprachliche, bildungssprachliche und mathematisch-fachsprachliche Register unterscheiden, die jeweils durch unterschiedliche sprachliche Merkmale bestimmt sind.

Die Bedingungen der Veränderungen von Sprache betreffen auch das Konzept des Registers. Der Wechsel von sprachlichen Ressourcen unterliegt ebenfalls dem Register und wird als Registervariation diskutiert. Registervariationen sind ein empirisches Phänomen, für das unterschiedliche Herangehensweise der Analyse existieren, wobei besonders computerbasierte Ansätze, die eine Kollektion von Texten (Korpus) verwenden, häufig genutzte Verfahren sind. Für die Analyse der sprachlichen Merkmale in einem Korpus existieren verschiedene Methoden. Es können die einzelnen sprachliche Merkmale in Form von Häufigkeitsanalysen betrachtet werden. Daneben existiert die Möglichkeit, das gemeinsame Vorkommen von sprachlichen Merkmalen zu betrachten. Als Methode zur Betrachtung des gemeinsamen Vorkommens von sprachlichen Merkmalen wird die Faktorenanalyse verwendet, die zur Gruppe der multivariaten Verfahren gezählt wird.

Ausblick: Das Register und die damit in Verbindung stehenden Konzepte erklären die Veränderungen von Sprache in der Nutzung. Sprachliche Variationen wie das schulsprachliche, bildungssprachliche und mathematisch-fachsprachliche Register können zu Hürden im Verstehen von Texten führen. Welche Grundlage diese Hürden im Textverstehen haben, wird im nachfolgenden Kapitel 5 erörtert. 
Open Access Dieses Kapitel wird unter der Creative Commons Namensnennung 4.0 International Lizenz (http://creativecommons.org/licenses/by/4.0/deed.de) veröffentlicht, welche die Nutzung, Vervielfältigung, Bearbeitung, Verbreitung und Wiedergabe in jeglichem Medium und Format erlaubt, sofern Sie den/die ursprünglichen Autor(en) und die Quelle ordnungsgemäß nennen, einen Link zur Creative Commons Lizenz beifügen und angeben, ob Änderungen vorgenommen wurden.

Die in diesem Kapitel enthaltenen Bilder und sonstiges Drittmaterial unterliegen ebenfalls der genannten Creative Commons Lizenz, sofern sich aus der Abbildungslegende nichts anderes ergibt. Sofern das betreffende Material nicht unter der genannten Creative Commons Lizenz steht und die betreffende Handlung nicht nach gesetzlichen Vorschriften erlaubt ist, ist für die oben aufgeführten Weiterverwendungen des Materials die Einwilligung des jeweiligen Rechteinhabers einzuholen. 